\title{
Synthesis of Potential Schistosomicides: New
}

\section{2-(Alkylamino)-1-Octanethiosulfuric Acids}

\author{
Marcus Luiz de Oliveira Penido, David Lee Nelson* \\ Departamento de Bioquímica-Imunologia, Instituto de \\ Ciências Biológicas, Caixa Postal 2486 \\ 30161 - Belo Horizonte, MG, Brasil
}

\section{Dorila Piló-Veloso}

\author{
Departamento de Química, Instituto de Ciências Exatas, \\ Universidade Federal de Minas Gerais \\ 31270 - Belo Horizonte, MG, Brasil
}

Received: August 28,1989; October 30,1989

\begin{abstract}
Quatro novos ácidos 2-(alquilamino)-1-octanotiossulfúricos (1) foram sintetizados a partir de 1-octeno. 1-Octeno foi epoxidado com MCPBA ou com um sistema de duas fases composto de $\mathrm{H}_{2} \mathrm{O}_{2}$, tungstato de sódio, ácido fosfórico, 1-octeno e um agente de transferência de fase. $A$ reação de 1,2-epoxioctano com aminas primárias forneceu 1-(alquilamino)-2-octanois, os quais foram convertidos aos respectivos bromidratos de $\mathrm{N}$-alquil-2-bromo-1-octanaminas pela reação com ácido bromídrico, seguida por tribrometo de fósforo. Finalmente, a substituição do íon brometo com tiosulfato de sódio foi acompanhada pelo rearranjo via um intermediário aziridino, resultando na formação do produto 1. Os intermediários e produtos finais foram submetidos a triagem para atividade contra infecção por Schistosoma mansoni; somente os produtos finais nos quais, o grupo $\mathrm{N}$-alquil foi sec-butil ou isopropil demonstraramse ativos.
\end{abstract}

Four new 2-(alkylamino)-1-octanethiosulfuric acids (1) were synthesized from 1-octene. 1-Octene was epoxidized with MCPBA or with a two-phase system composed of $\mathrm{H}_{2} \mathrm{O}_{2}$, sodium tungstate, phosphoric acid, 1-octene and a phase transfer agent. Reaction of the 1,2-epoxyoctane with primary amines furnished 1-(alkylamino)-2-octanols which were converted to the respective $\mathrm{N}$-alkyl-2-bromo-1-octanamine hydrobromides by reaction with hydrobromic acid, followed by phosphorus tribromide. Finally, substitution of the bromide ion with sodium thiosulfate was accompanied by rearrangement via an aziridine intermediate, resulting in formation of the product. 1. The intermediates and the final products were screened for activity against infection by Schistosoma mansoni, only the final products in which the N-alkyl group was sec-butyl or isopropyl exhibited activity.

Key words: 2-(alkylamino)-1-octanethiosulfuric acids; schistosomicides; Schistosoma mansoni.

\section{Introduction}

It has been demonstrated in our laboratories that certain 2-(alkylamino) ethanethiosulfuric acids ${ }^{1}$ present biological activity in mice experimentally infected with $S$. mansoni using the oogram method ${ }^{2}$. In order to obtain more information about the structure-activity relationship of this class of compounds, we undertook the synthesis of various 2-(alkylamino)-1-alkanethiosulfuric acids (1) bearing alkyl groups ( $\left.R^{\prime}\right)$ of various sizes alpha to the amino group:

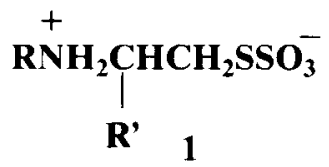

The present work reports the syntesis of four new compounds (1a-d) bearing n-hexyl groups bound to this position by the sequence of reactions shown in Scheme I. 
<smiles>C=CCCC(C)(C)C</smiles><smiles>C#CCCC(Br)C(CC(C)C)[Mg]Br</smiles>

5

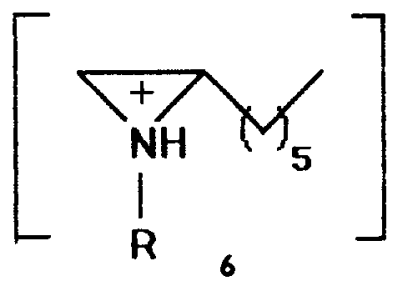

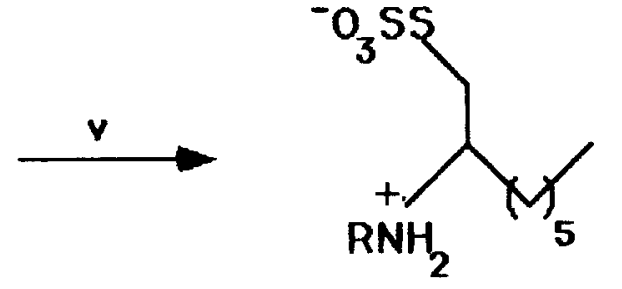

1

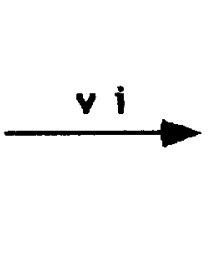

RNH

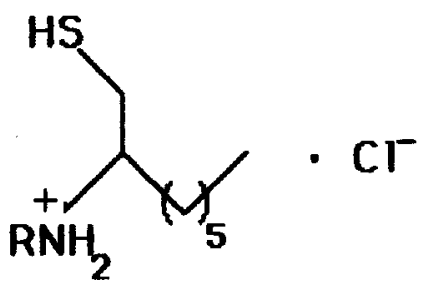

9

Scheme 1. Synthesis of 2-(alkylamino)-1-octanethiosulfuric acids (la-d). ia) $\mathrm{m}$-ClC $\mathrm{CH}_{6} \mathrm{CO}_{3} \mathrm{H}$; ib) $\mathrm{H}_{2} \mathrm{O}_{2} \mathrm{Na}_{2} \mathrm{WO}_{3}$, $\mathrm{H}_{3} \mathrm{PO}_{4}$, Aliquat 336; ii) $\mathrm{RNH}_{2} / \mathrm{MeOH}$; iii) $\mathrm{HBr}$ : iv) $\mathrm{PBr}_{3}$; v) $\mathrm{Na}_{2} \mathrm{~S}_{2} \mathrm{O}_{3} / \mathrm{H}_{2} \mathrm{O}-\mathrm{EtOH}$; vi) Conc. $\mathrm{HCl}$

\section{Experimental}

Infrared spectra were registered on a Perkin-Elmer model 283-B spectrophotometer. NMR spectra were determined on Varian model EM-360A and Brucker model A 80 instruments. Chemical shifts are reported as $\delta$ values in parts per million relative to tetramethylsilane $(\delta 0.00)$ as internal standard. Electron impact and chemical ionization mass spectra were determined on Finnigan 4000 and Varian MAT 311A mass spectrometers. Melting points were determined on a Bock-Monoscop Koffler type melting point apparatus and are uncorrected.

$m$-Chloroperbenzoic acid was prepared by classical methods $s^{3-5}$ from $m$-chlorobenzoic acid. All other reagents were obtained commercially and were of reagent grade.

1,2-epoxyoctane (3). Epoxidation of 1-octene (Aldrich) was performed utilizing either $m$-chloroperbenzoic acid ${ }^{6}$ or the catalytic system formed from sodium tungstate, phosphoric acid and hydrogen peroxide under phase transfer conditions according to the procedure of Venturello et $a l^{7}$, with yields of $70 \%$ and $50 \%$, respectively, based on the quantity of 1-octene used. $\mathrm{n}_{\mathrm{d}}{ }^{25}=1.3975 ;\left(\mathrm{Lit}^{23}\right.$ : $\left.\mathrm{n}_{\mathrm{d}}{ }^{20}=1.4197\right)$; bp $76^{\circ} \mathrm{C} / 30 \mathrm{mmHg}$; $\left(\mathrm{Lit}^{24}: 62-63^{\circ} \mathrm{C} / 17\right.$ $\mathrm{mmHg}$ ).

Synthesis of 1-(alkylamino)-2-octanols (4) - To a mixture of 0.3 mole of alkylamine and 0.1 mole of methanol in a round bottom flask equipped with a condenser and drying tube, was added 0.1 mole of 1,2-epoxyoctane dropwise with stirring at room temperature. When the addition was complete, the mixture was refluxed for $24 \mathrm{~h}$ (n- alkylamines) or $48 \mathrm{~h}$ (branched alkyl amines). The excess amine and methanol were distilled at atmospheric pressure. Liquid products were distilled under reduced pressure while solid products were recrystallized from methanol. The respective yields, melting or boiling points and spectral characteristics of the products are as follows:

I-(Butylamino)-2-octanol (4a). Yield $87 \% ; \mathrm{mp} 52{ }^{\circ} \mathrm{C} ; \mathrm{IR}$ (KBr) 3600, 3040, 2960, 2920, 2880, 2810, 1450, 1375, 1355, $1110,1085,990,885,855,710 \mathrm{~cm}^{-1}$; ${ }^{1} \mathrm{H}$ NMR $\left(\mathrm{CCl}_{4}\right) \delta$ $0.99(\mathrm{~m}, 6 \mathrm{H}), 1,45(\mathrm{~m}, 14 \mathrm{H}), 2.69(\mathrm{~m}, 4 \mathrm{H}), 2.81(\mathrm{~s}, 2 \mathrm{H})$, $3.55(\mathrm{~m}, 1 \mathrm{H})$; MS (EI) $\mathrm{m} / \mathrm{z}(\%):[\mathrm{M}+\mathrm{H}]^{+}=202(0.2)$, $\left[\mathrm{M}+\mathrm{H} \cdot \mathrm{H}_{2} \mathrm{O}\right]^{+}=184(0.05), \quad\left[\mathrm{n}-\mathrm{C}_{4} \mathrm{H}_{9} \mathrm{NHCH}_{2} \mathrm{CH}=\right.$ $\mathrm{OH}]^{+}=116(5), 87(5),\left[\mathrm{n}-\mathrm{C}_{4} \mathrm{H}_{9} \mathrm{NH}=\mathrm{CH}_{2}\right]^{+}=86(100)$, $\left[\mathrm{C}_{5} \mathrm{H}_{9}\right]^{+}=69(4),\left[\mathrm{C}_{4} \mathrm{H}_{9}\right]=57(14), 55(13),\left[\mathrm{C}_{2} \mathrm{H}_{6} \mathrm{~N}\right]^{+}$ $=44$ (88), 41 (32); $\mathrm{MS}(\mathrm{Cl}) \mathrm{m} / \mathrm{z}(\%):\left[\mathrm{M}+\mathrm{C}_{4} \mathrm{H}_{9}\right]^{+}=$ $258(4),\left[\mathrm{M}+\mathrm{C}_{4} \mathrm{H}_{9}-\mathrm{H}_{2} \mathrm{O}\right]^{+}=240(2),[\mathrm{M}+\mathrm{H}]^{+}=202$ (100), $\left[\mathrm{M}+\mathrm{H}-\mathrm{H}_{2} \mathrm{O}\right]^{+}=184(6), 116(0.6),\left[\mathrm{n}-\mathrm{C}_{4} \mathrm{H}_{9} \mathrm{NH}=\right.$ $\left.\mathrm{CH}_{2}\right]^{+}=86(17), 69$ (2). Anal. Calcd. for $\mathrm{C}_{12} \mathrm{H}_{27} \mathrm{NO}: \mathrm{C}$, 70.47; H, 13.42; N, 6.96. Found: C, 71.66; H, 13.70; N, 7.20 .

I-[(1-Methylpropyl)aminol]-2-octanol (4b). Yield 75\%; bp $95{ }^{\circ} \mathrm{C}(3 \mathrm{mmHg}) ; \mathrm{n}_{\mathrm{d}}{ }^{25}=1.4400 ;$ IR (liquid film) $3700-3020,3000-2600,1670,1460,1360,1350,1160,1120$, $1070,710 \mathrm{~cm}^{-1} ;{ }^{1} \mathrm{H} \mathrm{NMR}\left(\mathrm{CCl}_{4}\right) \delta 0.91(\mathrm{t}, \mathrm{J}=7 \mathrm{~Hz}, 6 \mathrm{H})$, $1.05(\mathrm{~d}, \mathrm{~J}=6 \mathrm{~Hz}, 3 \mathrm{~h}), 1.41(\mathrm{~m}, 12 \mathrm{H}), 2.50(\mathrm{~m}, 3 \mathrm{H}), 2.62$ $(\mathrm{s}, 2 \mathrm{H}), 3.49(\mathrm{~m}, 1 \mathrm{H})$; $\mathrm{MS}(\mathrm{EI}) \mathrm{m} / \mathrm{z}(\%):[\mathrm{M}+\mathrm{H}]^{+}=$ $202(9),\left[\mathrm{M}+\mathrm{H}_{-} \mathrm{H}_{2} \mathrm{O}\right]^{+}=184(0.3), 172(8), 154(2)$, [s$\left.\mathrm{C}_{4} \mathrm{H}_{9} \mathrm{NHCH}_{2} \mathrm{CH}=\mathrm{OH}\right]^{+}=116(4), 98(0.8),\left[\mathrm{s}-\mathrm{C}_{4} \mathrm{H}_{9} \mathrm{NH}=\right.$ $\left.\mathrm{CH}_{2}\right]^{+}=86(100), 72(7),\left[\mathrm{C}_{5} \mathrm{H}_{9}\right]^{+}=69(12), 60()$, $\left[\mathrm{C}_{4} \mathrm{H}_{9}\right]^{+}=57(22), 55(20),\left[\mathrm{C}_{2} \mathrm{H}_{6} \mathrm{~N}\right]^{+}=44$ (29); MS 
(Cl) $\mathrm{m} / \mathrm{z}(\%):\left[\mathrm{M}+\mathrm{C}_{4} \mathrm{H}_{9}\right]^{+}=258(5),\left[\mathrm{M}+\mathrm{C}_{4} \mathrm{H}_{9}-\mathrm{H}_{2} \mathrm{O}\right]^{+}$ $=240(2),[\mathrm{M}+\mathrm{H}]^{+}=202(100),\left[\mathrm{M}+\mathrm{H}-\mathrm{H}_{2} \mathrm{O}\right]^{+}=184$ (7), $172(3),\left[s-\mathrm{C}_{4} \mathrm{H}_{9} \mathrm{NH}=\mathrm{CH}_{2}\right]^{+}=86(13), 72$ (4).

1-[(1-Methylethyl)amino]-2-octanol (4c). Yield 73\%; bp $65^{\circ} \mathrm{C}$ ( $2 \mathrm{mmHg}$ ); $\mathrm{n}_{\mathrm{d}}{ }^{25}=1.4460$; IR (liquid film) 3700-302, $2960,2840,1650,1460,1380,1170,1120,1070,710 \mathrm{~cm}^{-1}$; ${ }^{1} \mathrm{H}$ NMR $\left(\mathrm{CCl}_{4}\right) \delta 0.97(\mathrm{~m}, 3 \mathrm{H}), 1.21(\mathrm{~d}, \mathrm{~J}=7 \mathrm{~Hz}, 6 \mathrm{H})$, $1.39(\mathrm{~m}, 10 \mathrm{H}), 2.61(\mathrm{~m}, 3 \mathrm{H}), 2.90(\mathrm{~s}, 2 \mathrm{H}), 3.52(\mathrm{~m}, 1 \mathrm{H})$; ${ }^{1} \mathrm{H}$ NMR $\left(\mathrm{CF}_{3} \mathrm{COOH}\right) \delta 0.92(\mathrm{t}, \mathrm{J}=7 \mathrm{~Hz}, 3 \mathrm{H}), 1.40(\mathrm{~m}$, $10 \mathrm{H}), 1.51$ (d, $\mathrm{J}=7 \mathrm{~Hz}, 6 \mathrm{H}), 3.47(\mathrm{~m}, 3 \mathrm{H}), 4.21(\mathrm{~m}, 1 \mathrm{H})$; MS (EI) m/z (\%): $[\mathrm{M}+\mathrm{H}]^{+}=188(46),\left[\mathrm{M}+\mathrm{H}-\mathrm{H}_{2} \mathrm{O}\right]^{+}$ $=170(2),\left[\left(\mathrm{CH}_{3}\right)_{2} \mathrm{CHNHCH}_{2} \mathrm{CH}=\mathrm{OH}\right]^{+}=102(7), 73$ (8), $\left[\left(\mathrm{CH}_{3}\right)_{2} \quad \mathrm{CHNH}=\mathrm{CH}_{2}\right]^{+}=72(100), 60$ (14), $\left[\mathrm{C}_{4} \mathrm{H}_{8}\right]^{+}=56(17),\left[\mathrm{C}_{2} \mathrm{H}_{6} \mathrm{~N}\right]^{+}=44(21) ; \mathrm{MS}(\mathrm{CI}) \mathrm{m} / \mathrm{z}$ (\%): $\left[\mathrm{M}+\mathrm{C}_{4} \mathrm{H}_{9}\right]^{+}=244(7),\left[\mathrm{M}+\mathrm{C}_{4} \mathrm{H}_{9}-\mathrm{H}_{2} \mathrm{O}\right]^{+}=226$ (3), $[\mathrm{M}+2 \mathrm{H}]=189(17),[\mathrm{M}+\mathrm{H}]^{+}=188(100),[\mathrm{M}+\mathrm{H}-$ $\left.\mathrm{H}_{2} \mathrm{O}\right]^{+}=170(8),\left[\left(\mathrm{CH}_{3}\right)_{2} \mathrm{CHNH}=\mathrm{CH}_{2}\right]^{+}=72(21), 60$ (2).

I-(Cyclohexylamino)-2-octanol (4d). Yield 55\%; mp $50^{\circ} \mathrm{C}$; IR (KBr) 3700 - 3040, 2980, 2840, 1660, 1440, 1360, $1100,1050,870,700 \mathrm{~cm}^{-1} ;{ }^{1} \mathrm{H}$ NMR $\left(\mathrm{CCl}_{4}\right) \delta 0.92(\mathrm{t}, \mathrm{J}=$ $7 \mathrm{~Hz}, 3 \mathrm{H}), 1.25(\mathrm{~m}, 16 \mathrm{H}), 1.78(\mathrm{~m}, 4 \mathrm{H}), 2.45(\mathrm{~m}, 3 \mathrm{H})$, $2.65(\mathrm{~s}, 2 \mathrm{H}), 3.52(\mathrm{~m}, 1 \mathrm{H}) ; \mathrm{MS}(\mathrm{EI}) \mathrm{m} / \mathrm{z}(\%):[\mathrm{M}+\mathrm{H}]^{+}$ $=228(5),\left[\mathrm{M}+\mathrm{H}-\mathrm{H}_{2} \mathrm{O}\right]^{+}=210(0.11), 184(1), 166(0.6)$, $\left[\mathrm{C}_{6} \mathrm{H}_{11} \mathrm{NHCH}_{2} \mathrm{CH}=\mathrm{OH}\right]^{+}=142$ (3), $126(0.2)$, $\left[\mathrm{C}_{6} \mathrm{H}_{11} \mathrm{NH}=\mathrm{CH}_{2}\right]^{+}=112(100),\left[\mathrm{C}_{6} \mathrm{H}_{11} \mathrm{NH}_{3}\right]^{+}=100(1)$, $\left[\mathrm{C}_{6} \mathrm{H}_{11}\right]^{+}=83(12),\left[\mathrm{C}_{5} \mathrm{H}_{10}\right]^{+}=70(7), 60(9),\left[\mathrm{C}_{4} \mathrm{H}_{7}\right]^{+}$ $=55$ (47), 44 (11); MS (CI) m/z (\%): $\left[\mathrm{M}+\mathrm{H}+\mathrm{C}_{4} \mathrm{H}_{9}\right]^{+}$ $=284(5),[\mathrm{M}+\mathrm{H}]^{+}=228(100),\left[\mathrm{M}+\mathrm{H}-\mathrm{H}_{2} \mathrm{O}\right]^{+}=210$ (6), $\left[\mathrm{C}_{6} \mathrm{H}_{11} \quad \mathrm{NHCH}_{2} \mathrm{CH}=\mathrm{OH}\right]^{+}=142(0.4), \quad\left[\mathrm{C}_{6} \mathrm{H}_{11}\right.$ $\left.\mathrm{NH}=\mathrm{CH}_{2}\right]^{+}=112(14),\left[\mathrm{C}_{6} \mathrm{H}_{11} \mathrm{NH}_{3}\right]^{+}=100(1)$.

Synthesis of $\mathrm{N}$-alkyl-2-bromo-1-octanamine hydrobromides (5) - Concentrated $\mathrm{HBr}(0.1$ mole) was added to 0.1 mole of aminooctanol 4 with stirring at $0{ }^{\circ} \mathrm{C}$. The precipitate was filtered, recrystallized from ethanol and dried over $\mathrm{P}_{2} \mathrm{O}_{5}$. A mixture of 0.1 mole of this hydrobromide, 0.05 mole $\mathrm{PBr}_{3}$ and $15 \mathrm{~m} \ell$ benzene was refluxed for three hours, the progress of the reaction being accompanied by TLC. The benzene and excess $\mathrm{PBr}_{3}$ were destilled under reduced pressure, the residue was washed with anhydrous ether and the resulting white crystalline precipitate was recrystallized from ethanol and chloroform. The yields, melting points and spectral data of the respective products are listed below.

N-butyl-2-bromo-I-octanamine hydrobromide (5a). Yield 88\%; mp 203-5 ${ }^{\circ} \mathrm{C}$; IR (KBr) 2960, 2920, 2860, 2870, $2410,1580,1450,1370,1300,710,530 \mathrm{~cm}^{-1} ;{ }^{1} \mathrm{H}$ NMR $\left(\mathrm{CDCl}_{3}\right) \delta 1.01(\mathrm{t}, \mathrm{J}=6 \mathrm{~Hz}, \mathrm{~Hz}, 6 \mathrm{H}), 1.42(\mathrm{~m}, 10 \mathrm{H}), 1.99(\mathrm{~m}$, $4 \mathrm{H}), 3.30(\mathrm{~m}, 4 \mathrm{H}), 4.62(\mathrm{~m}, 1 \mathrm{H}), 8.10-9.80(\mathrm{~m}, 2 \mathrm{H})$; MS (EI) $\mathrm{m} / \mathrm{z}(\%):\left[\mathrm{M}\left({ }^{81} \mathrm{Br}\right)-\mathrm{C}_{3} \mathrm{H}_{7}+\mathrm{H}\right]^{+}=223(0.2)$, $\left[\mathrm{M}\left({ }^{81} \mathrm{Br}\right)-\mathrm{C}_{3} \mathrm{H}_{7}\right]=222(2),\left[\mathrm{M}\left({ }^{79} \mathrm{Br}\right)-\mathrm{C}_{3} \mathrm{H}_{7}+\mathrm{H}\right]=221$ (0.35), $\left[\mathrm{M}\left({ }^{79} \mathrm{Br}\right)-\mathrm{C}_{3} \mathrm{H}_{7}\right]^{+}=220$ (2), $\left[\mathrm{n}-\mathrm{C}_{4} \mathrm{H}_{9} \mathrm{NH}=\right.$ $\left.\mathrm{CH}_{2}\right]^{+}=86(100), 69(11), 55$ (17), 44 (70), 41 (47); MS (CI) $\mathrm{m} / \mathrm{z}(\%):\left[\mathrm{M}\left({ }^{81} \mathrm{Br}\right)+\mathrm{C}_{4} \mathrm{H}_{9}\right]^{+}=322(1),\left[\mathrm{M}\left({ }^{79} \mathrm{Br}\right)+\right.$ $\left.\mathrm{C}_{4} \mathrm{H}_{9}\right]^{+}=320(1),\left[\mathrm{M}\left({ }^{81} \mathrm{Br}\right)+\mathrm{C}_{3} \mathrm{H}_{3}\right]^{+}=304(2),\left[\mathrm{M}\left({ }^{79} \mathrm{Br}\right)\right.$ $\left.+\mathrm{C}_{3} \mathrm{H}_{3}\right]^{+}=302(2),\left[\mathrm{M}\left({ }^{81} \mathrm{Br}\right)+2 \mathrm{H}\right]=267(12)$, $\left[\mathrm{M}\left({ }^{81} \mathrm{BR}\right)+\mathrm{H}\right]=266(87),\left[\mathrm{M}\left({ }^{79} \mathrm{Br}\right)+2 \mathrm{H}\right]=265(15)$, $\left[\mathrm{M}\left({ }^{79} \mathrm{Br}\right)+\mathrm{H}\right]=264(100),[\mathrm{M}+2 \mathrm{H}-\mathrm{HBr}]^{+}=185(7)$, $[\mathrm{M}-\mathrm{HBr}]^{+}=184(57),\left[\mathrm{n}-\mathrm{C}_{4} \mathrm{H}_{9} \mathrm{NH}=\mathrm{CH}_{2}\right]^{+}=86(24)$.

Anal. Calcd. for $\mathrm{C}_{12} \mathrm{H}_{27} \mathrm{NBr}_{2}: \mathrm{C}, 41.66 ; \mathrm{H}, 8.09 ; \mathrm{N}$, 4.04. Found $C, 41.74 ; \mathrm{H}, 8.07 ; \mathrm{N}, 4.20$.
N-(I-Methylpropyl)-2-bromo-1-octanamine hydrobromide (5b). Yield 77\%; mp $121-4{ }^{\circ} \mathrm{C}$; IR (KBr) 2950, 2920, 2840, 2730, 2400,1560,1440,1400,1310,520 $\mathrm{cm}^{-1} ;{ }^{1} \mathrm{H}$ NMR $\left(\mathrm{CDCl}_{3}\right) \delta 1.09(\mathrm{t}, \mathrm{J}=7 \mathrm{~Hz}, 6 \mathrm{H}), 1.37(\mathrm{~m}, 8 \mathrm{H})$, $1.55(\mathrm{~d}, \mathrm{~J}=6 \mathrm{~Hz}, 3 \mathrm{H}), 2.01(\mathrm{~m}, 4 \mathrm{H}), 3.45(\mathrm{~m}, 3 \mathrm{H}), 4.69$ (m, 1H), 8.2-9.9 (m, 2H); MS (EI) m/z (\%): [M( $\left.{ }^{81} \mathrm{Br}\right)-$ $\left.\mathrm{CH}_{3}\right]^{+}=250(1),\left[\mathrm{M}\left({ }^{79} \mathrm{Br}\right)-\mathrm{CH}_{3}\right]^{+}=248(1),\left[\mathrm{M}\left({ }^{81} \mathrm{Br}\right)-\right.$ $\left.\mathrm{C}_{2} \mathrm{H}_{5}\right]^{+}=236(16),\left[\mathrm{M}\left({ }^{79} \mathrm{Br}\right)-\mathrm{C}_{2} \mathrm{H}_{5}\right]^{+}=234$ (17), [M$\mathrm{HBr}+\mathrm{H}]^{+}=184(2),\left[\mathrm{M}-\mathrm{HBr}-\mathrm{CH}_{3}\right]^{+}=168(2),[\mathrm{M}-$ $\left.\mathrm{HBr}-\mathrm{C}_{2} \mathrm{H}_{5}\right]^{+}=154(18),\left[\mathrm{M}-\mathrm{HBr}-\mathrm{C}_{3} \mathrm{H}_{7}\right]^{+}=140(3),[\mathrm{M}-$ $\left.\mathrm{HBr}-\mathrm{C}_{4} \mathrm{H}_{9}\right]^{+}=126(8),\left[\mathrm{M}-\mathrm{HBr}-\mathrm{C}_{5} \mathrm{H}_{11}\right]^{+}=112(4),[\mathrm{M}-$ $\left.\mathrm{HBr}-\mathrm{C}_{6} \mathrm{H}_{13}\right]^{+}=98(8),\left[\mathrm{s}-\mathrm{C}_{4} \mathrm{H}_{9} \mathrm{NH}=\mathrm{CH}_{2}\right]^{+}=86(29)$, $69(12), 56(26), 44(48),\left[\mathrm{C}_{3} \mathrm{H}_{5}\right]^{+}=41(100)$. MS (CI) $\mathrm{m} / \mathrm{z}(\%):\left[\mathrm{M}\left({ }^{81} \mathrm{Br}\right)-\mathrm{C}_{4} \mathrm{H}_{9}\right]=322(0.8)\left[\mathrm{M}\left({ }^{79} \mathrm{Br}\right)+\mathrm{C}_{4} \mathrm{H}_{9}\right]^{+}$ $=320(0.8),\left[\mathrm{M}\left({ }^{81} \mathrm{Br}\right)+\mathrm{C}_{3} \mathrm{H}_{3}\right]^{+}=304(2),\left[\mathrm{M}\left({ }^{79} \mathrm{Br}\right)+\right.$ $\left.\mathrm{C}_{3} \mathrm{H}_{3}\right]^{+}=302(2),\left[\mathrm{M}\left({ }^{81} \mathrm{Br}\right)+2 \mathrm{H}\right]^{+}=267(13),\left[\mathrm{M}\left({ }^{81} \mathrm{Br}\right)\right.$ $+\mathrm{H}]^{+}=266(94),\left[\mathrm{M}\left({ }^{79} \mathrm{Br}\right)+2 \mathrm{H}\right]^{+}=265(15),\left[\mathrm{M}\left({ }^{79} \mathrm{Br}\right)\right.$ $+\mathrm{H}]^{+}=264(100),\left[\mathrm{M}\left({ }^{81} \mathrm{Br}\right)-\mathrm{C}_{2} \mathrm{H}_{5}\right]^{+}=236(5),\left[\mathrm{M}\left({ }^{79} \mathrm{Br}\right)-\right.$ $\left.\mathrm{C}_{2} \mathrm{H}_{5}\right]^{+}=234(5),[\mathrm{M}+2 \mathrm{H}-\mathrm{HBr}]^{+}=185(8),\left[\mathrm{M}+\mathrm{H}_{-}\right.$ $\mathrm{HBr}]^{+}=184(65),\left[\mathrm{s}-\mathrm{C}_{4} \mathrm{H}_{9} \mathrm{NH}=\mathrm{CH}_{2}\right]^{+}=86(6)$.

$\mathrm{N}$-(1-Methylethyl)-2-bromo-1-octanamine hydrobromide (5c). Yield 80\%; mp 122-4 ${ }^{\circ} \mathrm{C}$; IR (KBr) 2940, 2850, 2790, $2540,2420,1580,1450,1380,1270,1150,1130,990,960$, $720,530 \mathrm{~cm}^{-1} ;{ }^{1} \mathrm{HNMR}\left(\mathrm{CDCl}_{3}\right) \delta 0.91(\mathrm{t}, \mathrm{J}=6 \mathrm{~Hz}, 3 \mathrm{H})$, $1.39(\mathrm{~m}, 8 \mathrm{H}), 1.65(\mathrm{~d}, \mathrm{~J}=7 \mathrm{~Hz}, 6 \mathrm{H}), 1.99(\mathrm{~m}, 2 \mathrm{H}), 3.60$ $(\mathrm{m}, 3 \mathrm{H}), 4.70(\mathrm{~m}, 1 \mathrm{H}), 8.2-10.1(\mathrm{~m}, 2 \mathrm{H}) ; \mathrm{MS}(\mathrm{EI}) \mathrm{m} / \mathrm{z}(\%)$ : $\left[\mathrm{M}\left({ }^{81} \mathrm{Br}\right)-\mathrm{CH}_{3}\right]^{+.}=236(1),\left[\mathrm{M}\left({ }^{79} \mathrm{Br}\right)-\mathrm{CH}_{3}\right]^{+\cdot}=234(2)$, $[\mathrm{M}-\mathrm{H}-\mathrm{HBr}]^{+.}=170(2),\left[\mathrm{M}-\mathrm{C}_{6} \mathrm{H}_{13}\right]^{+\cdot}=164(0.4),[\mathrm{M}-$ $\left.\mathrm{HBr}-\mathrm{C}_{4} \mathrm{H}_{9}\right]^{+}=112(1),\left[\left(\mathrm{CH}_{3}\right)_{2} \mathrm{CH}=\mathrm{NHCH}_{2}\right]^{+}=72$ (100); MS (CI) m/z (\%); $\left[\mathrm{M}\left({ }^{81} \mathrm{Br}\right)+\mathrm{C}_{4} \mathrm{H}_{9}\right]^{+}=308(1)$, $\left[\mathrm{M}\left({ }^{79} \mathrm{Br}\right)+\mathrm{C}_{4} \mathrm{H}_{9}\right]^{+}=306(0.6),\left[\mathrm{M}\left({ }^{81} \mathrm{Br}\right)+\mathrm{C}_{3} \mathrm{H}_{7}\right]^{+}=$ 294(0.5), $\left[\mathrm{M}\left({ }^{79} \mathrm{Br}\right)+\mathrm{C}_{3} \mathrm{H}_{7}\right]^{+}=292(0.7),\left[\mathrm{M}\left({ }^{81} \mathrm{Br}\right)+\right.$ $\left.\mathrm{C}_{3} \mathrm{H}_{5}\right]^{+}=290(2),\left[\mathrm{M}\left({ }^{79} \mathrm{Br}\right)+\mathrm{C}_{3} \mathrm{H}_{5}\right]^{+}=288(2),[\mathrm{M}$ $\left.\left({ }^{81} \mathrm{Br}\right)+2 \mathrm{H}\right]^{+}=253(11),\left[\mathrm{M}\left({ }^{81} \mathrm{Br}\right)+\mathrm{H}\right]^{+}=252(90)$, $\left[\mathrm{M}\left({ }^{79} \mathrm{Br}\right)+2 \mathrm{H}\right]^{+}=250(100),\left[\mathrm{M}-\mathrm{HBr}+\mathrm{C}_{3} \mathrm{H}_{7}\right]^{+}=226$ (0.6), $[\mathrm{M}-\mathrm{HBr}+2 \mathrm{H}]^{+}=171(9),[\mathrm{M}-\mathrm{HBr}+\mathrm{H}]^{+}=170$ (81), $156(0.8), 128(1), 112(0.5), 100(6),\left[\left(\mathrm{CH}_{3}\right)_{2} \mathrm{CH}=\right.$ $\left.\mathrm{NHCH}_{2}\right]^{+}=72(38)$.

N-Cyclohexyl-2-bromo-1-octanamine hydrobromide (5d). Yield 60\%; mp 163-5 ${ }^{\circ} \mathrm{C}$; IR (KBr) 2920, 2870, 2760, $2740,2610,2540,2400,1580,1450,1400,720,530 \mathrm{~cm}^{-1}$; ${ }^{1} \mathrm{H}$ NMR $\left(\mathrm{CDCl}_{3}\right) \delta 0.95(\mathrm{t}, \mathrm{J}=6 \mathrm{~Hz}, 3 \mathrm{H}), 1.37(\mathrm{~m}, 8 \mathrm{H})$, $1.82(\mathrm{~m}, 6 \mathrm{H}), 2.30(\mathrm{~m}, 4 \mathrm{H}), 3.45(\mathrm{~m}, 3 \mathrm{H}), 4.61(\mathrm{~m}, 1 \mathrm{H})$, 8.2-9.7 (m, 2H); MS (EI) m/z (\%): $\left[\mathrm{M}\left({ }^{81} \mathrm{Br}\right)-\mathrm{C}_{3} \mathrm{H}_{7}\right]^{+}=$ $248(4),\left[\mathrm{M}\left({ }^{79} \mathrm{Br}\right)-\mathrm{C}_{3} \mathrm{H}_{7}\right]^{+}=246(6),\left[\mathrm{M}\left({ }^{79} \mathrm{Br}\right)-\mathrm{C}_{5} \mathrm{H}_{11}\right]^{+}=$ $218(0.2),[\mathrm{M}-\mathrm{HBr}-\mathrm{H}]^{+}=210(4),\left[\mathrm{M}-\mathrm{HBr}-\mathrm{C}_{3} \mathrm{H}_{7}\right]^{+}=$ 166 (3), 152 (2), 138 (2), 124(4), $\left[\mathrm{C}_{6} \mathrm{H}_{11} \mathrm{NH}=\mathrm{CH}_{2}\right]^{+}=$ 112(100), 96(5), 69(11), 41 (63); MS (CI) m/z (\%): $\left[\mathrm{M}\left({ }^{81} \mathrm{Br}\right)+2 \mathrm{H}\right]^{+}=293(13),\left[\mathrm{M}\left({ }^{81} \mathrm{Br}\right)+\mathrm{H}\right]^{+}=292(88)$, $\left[\mathrm{M}\left({ }^{79} \mathrm{Br}\right)+2 \mathrm{H}\right]^{+}=291(18),\left[\mathrm{M}\left({ }^{79} \mathrm{Br}\right)+2 \mathrm{H}\right]^{+}=290$ (100), $[\mathrm{M}-\mathrm{HBr}-\mathrm{H}]^{+}=210(91),\left[\mathrm{C}_{6} \mathrm{H}_{11} \mathrm{NH}=\mathrm{CH}_{2}\right]^{+}=$ 112(36), 100(4), 81(12), 69(20).

Synthesis of 2-(alkylamino)-1-octanethiosulfuric acids (1) - A solution containing 50 mmole of the bromooc- 
tylamine hydrobromide (5) and 50 mmole sodium thiosulfate pentahydrate in $15 \mathrm{mP} 50 \%$ ethanol was refluxed for 2.5 to $3 \mathrm{hs}$. The progress of the reaction was followed by TLC, using $0.5 \% \mathrm{PdCl}_{2}$ in $0.1 \mathrm{~N} \mathrm{HCl}$ to visualize the plates. Upon cooling a white crystalline product formed which was filtered and recrystallized from methanol. The yields, decomposition points and spectral data for the respective products are listed below:

2-(Butylamino)-1-octanethiosulfuric acid (1a). Yield $60 \%$; mp 234-7 ${ }^{\circ} \mathrm{C}$ (d); IR (KBr) 3140, 2960, 2840, 1580, $1460,1240,1190,102,630,520 \mathrm{~cm}^{-1}$; ${ }^{1} \mathrm{H}$ NMR $(60 \mathrm{MHz}$, $\left.\mathrm{CF}_{3} \mathrm{COOH}\right) \delta 1.11(\mathrm{t}, \mathrm{J}=7 \mathrm{~Hz}, 6 \mathrm{H}), 1,42(\mathrm{~m}, 8 \mathrm{H})$, 1.79(m,6H), $3.38(\mathrm{~m}, 3 \mathrm{H}), 3.68(\mathrm{~m}, 2 \mathrm{H}), 6.4-8.2(\mathrm{~m}, 2 \mathrm{H})$; ${ }^{1} \mathrm{H}$ NMR $\left(80 \mathrm{MHz}, \mathrm{D}_{2} \mathrm{O} / \mathrm{NaOH}\right) \delta 0.85(\mathrm{~m}, 6 \mathrm{H}), 1.26$ $(\mathrm{m}, 14 \mathrm{H}), 2.53(\mathrm{t}, \mathrm{J}=5.4 \mathrm{~Hz}, 2 \mathrm{H}) 2.91(\mathrm{~m}, 1 \mathrm{H}), 3.14(\mathrm{~m}, 2 \mathrm{H})$; MS (EI) m/z (\%): 218 (2), 217 (2), 216 (2), 184 (2), [n$\left.\mathrm{C}_{4} \mathrm{H}_{9} \mathrm{NH}=\mathrm{CH}\left(\mathrm{C}_{6} \mathrm{H}_{13}\right)\right]^{+}=170(100), 114(1), 86(2), 74(1)$, 71(8), 64(13), 55 (10); MS (CI) m/z (\%): 433 (7), 274 (13), $\left[\mathrm{n}-\mathrm{C}_{4} \mathrm{H}_{9} \mathrm{NHCH}\left(\mathrm{C}_{6} \mathrm{H}_{13}\right) \mathrm{CH}_{2} \mathrm{SH}+\mathrm{H}\right]^{+.}=218(100)$, 217(2), 216(19), 184(56), $170(18), 145$ (22), 129(4), 114(2), 86(1), 74 (55), 71 (9), 69 (12).

Anal.Calcd for $\mathrm{C}_{12} \mathrm{H}_{27} \mathrm{NO}_{3} \mathrm{~S}_{2}: \mathrm{C}, 48.48 ; \mathrm{H}, 9.09 ; \mathrm{N}, 4.71$. Found C, 48.52; H, 9.19; N, 4.32.

2-[(1-Methylpropyl)amino]-1-octanethiosulfuric acid (1b). Yield 50\%; mp $193-5^{\circ} \mathrm{C}$ (d); IR (KBr) 3020, 2960, 2920, 2840,1560,1450,1240,1190,1010, 620,520 cm${ }^{1} \mathrm{H}$ NMR $\left(60 \mathrm{MHz}, \mathrm{CF}_{3} \mathrm{COOH}\right) \delta 1.09(\mathrm{~m}, 6 \mathrm{H}), 1.45(\mathrm{~m}$, $9 \mathrm{H}), 1.85(\mathrm{~m}, 6 \mathrm{H}), 3.48(\mathrm{~m}, 2 \mathrm{H}), 3.70(\mathrm{~m}, 2 \mathrm{H}), 6.1-8.1$ (m, 2H); MS (EI) m/z (\%): $433(0,5) 218$ (3), 217 (5), 216 (4), 184 (2), $\left[\mathrm{s}-\mathrm{C}_{4} \mathrm{H}_{9} \mathrm{NH}=\mathrm{CH}\left(\mathrm{C}_{6} \mathrm{H}_{13}\right)\right]^{+}=170(100), 114$ (9), 86 (8), 74 (3) 71 (2), 64 (26), 55 (20); MS (Cl) m/z (\%): 433 (67), 274 (19), [s- $\mathrm{C}_{4} \mathrm{H}_{9} \mathrm{NHCH}\left(\mathrm{C}_{6} \mathrm{H}_{13}\right) \mathrm{CH}_{2} \mathrm{SH}$ $+\mathrm{H}]^{+}=218(100), 217(10), 216(47), 184(29), 170(36)$, 145 (13), 129 (6), 114(2), 86 (4), 74 (24), 71 (11), 69 (13).

Anal. Calcd. for $\mathrm{C}_{12} \mathrm{H}_{27} \mathrm{NO}_{3} \mathrm{~S}_{3}: \mathrm{C}, 48.48 ; \mathrm{H}, 9.09 ; \mathrm{N}$, 4.71. Found: C, 47.44; H, 9.20; N, 5.15.

2-[(1-Methylethyl)amino]-1-octanethiosulfuric acid (1c). Yield $60 \% \mathrm{mp} 212-5^{\circ} \mathrm{C}$ (d); IR (KBr) $3020,2980,2920$, $2840,1610,1460,1230,1190,1010,630,520 \mathrm{~cm}^{-1} ;{ }^{1} \mathrm{H}$ NMR $\left(60 \mathrm{MHz}, \mathrm{CF}_{3} \mathrm{COOH}\right) \delta 1.00(\mathrm{~m}, 3 \mathrm{H}), 1.58(\mathrm{~m}$, $12 \mathrm{H}), 1.92(\mathrm{~m}, 4 \mathrm{H}), 3.78(\mathrm{~m}, 4 \mathrm{H}) 6.3-7.8(\mathrm{~m}, 2 \mathrm{H})$; $\mathrm{MS}$ (EI) m/z (\%): 204 (1), 203 (4), $202(4), 170(2),\left[\left(\mathrm{CH}_{3}\right)_{2}\right.$ $\left.\mathrm{CHNH}=\mathrm{CH}\left(\mathrm{C}_{6} \mathrm{H}_{13}\right)\right]^{+}=156(100), 114(7), 72(10), 64$ (23), 60 (2), 58 (13), 57 (3), 55 (20); MS (CI) m/z (\%): 405 (41), 260 (16), [( $\left.\mathrm{CH}_{3}\right)_{2} \mathrm{CHNHCH}\left(\mathrm{C}_{6} \mathrm{H}_{13}\right) \mathrm{CH}_{2} \mathrm{SH}+$ $\mathrm{H}^{+}=204(100) 202(5), 170(22), 156(14), 129(0,4)$, 114(1) 72 (3), 60 (20).

Anal. Calcd. for $\mathrm{C}_{11} \mathrm{H}_{25} \mathrm{NO}_{3} \mathrm{~S}_{2}: \mathrm{C}, 46.60 ; \mathrm{H}, 8.83 ; \mathrm{N}$, 4.94. Found: C, 44.68; H, 8.72; N, 5.03.

2-(Cyclohexylamino)-1- octanethiosulfuric acid (1d). Yield 60\%; mp 231-4 ${ }^{\circ} \mathrm{C}$ (d); IR (KBr) 3000, 2920, 2860, $1610,1450,1260,1240,1180,1160,1010,630,520 \mathrm{~cm}^{-1}$; ${ }^{1} \mathrm{H}$ NMR $\left(60 \mathrm{MHz}, \mathrm{CF}_{3} \mathrm{COOH}\right) \delta 0.99(\mathrm{~m}, 3 \mathrm{H}), 1.41(\mathrm{~m}$,
12H), $1.94(\mathrm{~m}, 8 \mathrm{H}), 3.47(\mathrm{~m}, 2 \mathrm{H}), 3.72(\mathrm{~m}, 2 \mathrm{H}), 6.3-7.8$ $(\mathrm{m}, 2 \mathrm{H}) ;{ }^{1} \mathrm{H}$ NMR $\left(80 \mathrm{MHz}, \mathrm{CDCl}_{3} / \mathrm{NaOH}\right) \delta 0.80(\mathrm{~m}$, $3 \mathrm{H}), 1.22(\mathrm{~m}, 12 \mathrm{H}), 1.66(\mathrm{~m}, 8 \mathrm{H}), 2.43(\mathrm{~m}, 1 \mathrm{H}), 2.87(\mathrm{~m}$, 1H), $3.04(\mathrm{~m}, 2 \mathrm{H})$; MS (EI) $\mathrm{m} / \mathrm{z}(\%)$ : $485(0.3), 244$ (2), 243 (4), 242 (3), $\left.210(2),\left[\mathrm{C}_{6} \mathrm{H}_{11} \mathrm{NH}=\mathrm{CHC}_{6} \mathrm{H}_{13}\right)\right]^{+}=196$ (100), 114 (7), $112(1), 100(0,5), 64(10), 55$ (19); MS (CI) m/z (\%): 485 (63), $\left.300(27),\left[\mathrm{C}_{6} \mathrm{H}_{11} \mathrm{NHCHC}_{6} \mathrm{H}_{13}\right) \mathrm{CH}_{2} \mathrm{SH}+\mathrm{H}\right)^{+}$ $=244$ (100), 243 (9) 242 (42), 210 (24), 196 (22), 145 (9), 129 (6), 114 (1), 112 (0.6), 100 (23), 64 (10), 97 (0.4).

Anal.Calcd. for $\mathrm{C}_{14} \mathrm{H}_{29} \mathrm{NO}_{3} \mathrm{~S}_{2}: \mathrm{C}, 52.01 ; \mathrm{H}, 8.97 ; \mathrm{N}$, 4.33. Found: C, 50.60; H, 8.98; N, 4.57 .

Synthesis of N-butyl-1-mercapto-2-octanamine hydrochloride (9) - A mixture of 0.02 moles of 1a, 0.02 moles of $37 \% \mathrm{HC} \ell$ and $10 \mathrm{~m} \ell$ methanol was heated under reflux for $16 \mathrm{hr}$. The solvent was evaporated and the oily residue was treated with conc. $\mathrm{HCl}$ to form a white precipitate which was recrystallized from methanol. Yield $40 \%$; mp $163-5{ }^{\circ} \mathrm{C}$; IR (KBr) $3100-2600,2450,1580,1450,1380$, $1110,1030,720,480 \mathrm{~cm}^{-1}$; $1 \mathrm{H}$ NMR $\left(60 \mathrm{MHz}, \mathrm{CDC}_{3}\right)$ $\delta 0.99(\mathrm{t}, \mathrm{J}=6 \mathrm{~Hz}, 6 \mathrm{H}), 1.41(\mathrm{~m}, 10 \mathrm{H}), 1.97(\mathrm{~m}, 4 \mathrm{H}), 2.73$ $(\mathrm{m}, 1 \mathrm{H}), 3.02(\mathrm{~m}, 3 \mathrm{H}), 3.57(\mathrm{~m}, 2 \mathrm{H}), 8.9$ - $9.9($ broad $\mathrm{m}$, 2H). MS (EI) m/z (\%): 218 (2), 217 (2), $216(1), 184(8)$, $\left[\mathrm{n}-\mathrm{C}_{4} \mathrm{H}_{9} \mathrm{NH}=\mathrm{CH}\left(\mathrm{C}_{6} \mathrm{H}_{13}\right)\right]^{+}=170(100), 114(3), 86(18)$, 84 (14), 74 (14), 72 (16), 62 (25), 55 (38), 45 (43).

\section{Results}

1-Octene (2) was converted to the 1,2-epoxyoctane (3) by epoxidation with $m$-chloroperbenzoic acid ${ }^{6}$ or by reaction with the catalytic system composed of hydrogen peroxide, sodium tungstate and phosphoric acid, using Aliquat 336 as the phase transfer agent ${ }^{7}$. Although the latter system furnishes $80 \%$ yields based on the quantity of hydrogen peroxide utilized ${ }^{7}$, the yield based on 1 -octene is $50 \%$. The unreacted alkene was separated by distillation and re-used.

Treatment of 3 with an excess of primary amine ${ }^{8}$ furnished the amino-alcohol 4 . The ring opening occurs with regiospecific attack of the amine on the least substituted carbon $^{9-11}$. The amino-alcohol was converted to its hydrobromide salt to protect the amino group and subsequently treated with phosphorus tribromide to produce the bromoalkylamine $\mathbf{5}^{\mathbf{1 2}}$. Benzene was used as solvent in this step to reduce the probability of rearrangement, a problem which has been observed in the reaction of branched amino-alcohols ${ }^{13}$ with $\mathrm{PBr}_{3}$. No secondary products due to elimination were observed in this step. Finally, nucleophilic substitution by thiosulfate in $\mathbf{5}$ resulted in the formation of the 2-(alkylamino)-1-alkanethiosulfuric acids $(\mathbf{1})^{14}$. This product must arise from a rearrangement involving the formation an aziridine intermediate 6 which is then attaked by thiosulfate ion ${ }^{15-16}$. This rearrangement occurred in spite of the fact that the reaction was carried out under acidic conditions under which the amino group should be protonated. Racemic products were obtained in all cases. In the case of compounds $\mathbf{1 b}$, $\mathbf{4 b}$ and $\mathbf{5 b}$, a mixture of diasterisomers composed of two enantiomeric pairs was formed. No attempt was made to separate these isomers prior to biological assay.

Evidence for the assigned structures 1a-d is given prin- 
cipally by their mass spectra. The thiosulfuric acids are thermally unstable, decomposing above $100^{\circ} \mathrm{C}$ to form disulfides, thiols, sulfates and sulfur dioxide ${ }^{17}$ Therefore, molecular ion peaks corresponding to the amino alkanethiosulfuric acids are not observed in the mass spectra of these compounds, but one rather finds peaks corresponding to the disulfides, thiols and other decomposition products ${ }^{18-19}$. In the electron impact mass spectra of the intermediates 4 and 5 , the base peak corresponds to the fragment 7 (Scheme 2) which results from cleavage of the bond beta to the amino group. This is also a major peak in the chemical ionization spectra of these compounds. In the mass spectra of the thiosulfuric acids 1ad, a similar fragmentation (beta to the alkylamino group) gives rise to peaks corresponding to $\mathbf{8}$, as wel as other fragments that could arise only from the 2-(alkylamino)-1octanethiosulfuric acid isomer.

\section{$\mathrm{C}_{4} \mathrm{H}_{9} \mathrm{NHCH}_{2}{ }_{\mid}^{\mathrm{CH}}\left(\mathrm{CH}_{2}\right)_{5} \mathrm{CH}_{3}$}

10

In order to further confirm the identity of the thiosulfuric acid products, 1a was converted to the corresponding thiol (9) by acid hydrolysis. The mass spectrum of the product was analogous to that obtained for compounds 1a-d and different from that obtained by Vieira Filho and coworkers $^{20}$ for the N-butyl-2-mercapto-1-octanamine (10), whose mass spectrum is similar to those obtained for the intermediates 4 and 5 .

Finally, the intermediates $\mathbf{4}$ and 5 and the products 1ad were submitted for screening for curative activity in mice experimentally infected with $S$. mansoni, using the oogram method ${ }^{2,21-22}$. The results of these tests are shown in Table 1 .
Table 1. Activity of

2-(alkylamino)-1-octanethiosulfuric acids in mice previously infected by Schistosoma mansoni.

\begin{tabular}{lccl}
\hline $\begin{array}{c}\text { Compound } \\
(\mathrm{mg} / \mathrm{kg} / \text { day } \times 5 \text { days })\end{array}$ & $\begin{array}{c}\text { Dose p.o. } \\
(\text { Total/dead })\end{array}$ & Results \\
\hline 1a & 600 & $5 / 2$ & Inactive \\
1b & 300 & $5 / 0$ & Active $2 / 5$ \\
1c & 300 & $5 / 0$ & Active $4 / 5$ \\
1d & 300 & $5 / 0$ & In active \\
\hline
\end{tabular}

Legends for strutures and Schemes:
1a, $\mathbf{R}=$ n-butyl
1b, $\mathrm{R}=$ sec-butyl
1c, $\mathrm{R}=$ isopropyl
1d, $\mathbf{R}$ = cyclohexyl

\section{Conclusions}

With the above synthetic scheme, it was possible to obtain four new 2-(alkylamino)-1-octanethiosulfuric acids in good yield. In preliminary screening of new drugs, the intermediate amino-alcohols and bromoalkylamines (4 and 5) did not show activity, whereas, of the four aminoalkanethiosulfuric acids obtained, compounds $\mathbf{1 b}$ and 1c were observed to be active.

\section{Acknowledgements}

This work was supported by the Conselho Nacional de Desenvolvimento Científico e Tecnológico - CNPq and by the Financiadora de Estudos e Projetos - FINEP (Funtec.). Professors Nelson and Piló-Veloso were recipients of $\mathrm{CNPq}$ research fellowships and $\mathrm{Mr}$. Penido received a graduate scholarship furnished by the Coordenação de Aperfeiçoamento de Pessoal de Nivel Superior - CAPES. We wish to thank the Campus-wide Mass Spectrometry Center, Chemistry Department, Purdue University and the Instituto de Química, UNICAMP for determination
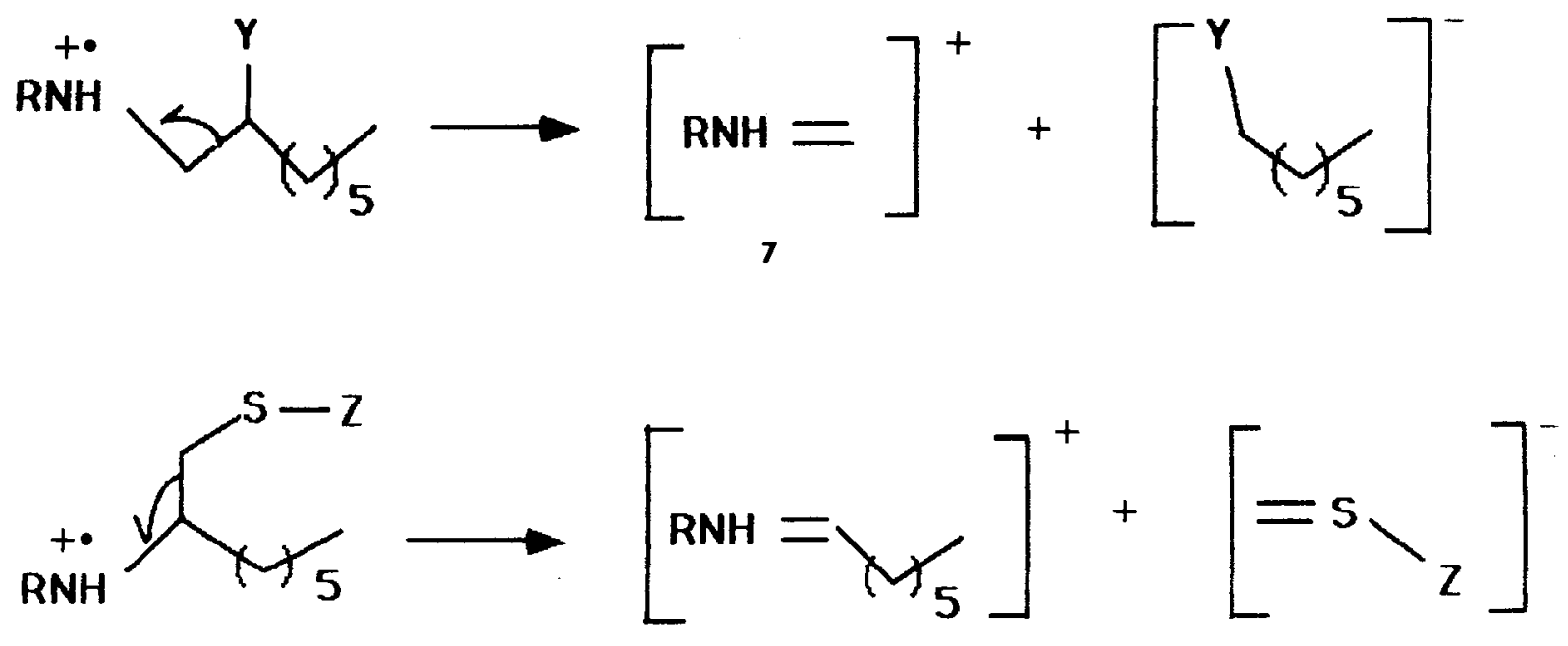

8

Scheme 2. Principal fragmentations of 1-(alkylamino)-2-octanols (4), bromides (5) and 2-(alkylaminol)-l-octanethiosulfuric acids (1 and its thermal decomposition products). $Y=O H, B r ; Z=S O_{3} H, H, S R$. 
of the mass spectra, as well as the Fundação Centro Tecnológico de Minas Gerais - CETEC and the Departamento de Química - Universidade de São Paulo for microanalyses. Biological screening was performed by the Grupo Interdepartamental de Estudos Sobre Esquitossomose GIDE - UFMG. Infrared and NMR spectra were determined by the Departamento de Química, Instituto de Ciências Exatas, Universidade Federal de Minas Gerais and the Departamento de Quimica, Universidade de Brasilia.

\section{References}

1. D.L. Nelson and J. Pellegrino, Rev. Inst. Med. Trop. São Paulo 18, 365-370 (1976).

2. J. Pellegrino and N. Katz, Adv. Parasitol. 6, 233-260 (1968).

3. L.F. Fieser and M. Fieser, Reagents for Organic Synthesis (J. Wiley \& Sons, New York, 1967), pp. 135-139.

4. R.M. Herbst and K.R. Wilson, J. Org. Chem. 22 , 1142-1145 (1957)

5. R.N. McDonald, R.N. Steppel and J. E Dorsey, In: R. Breslow (Eds.) Org. Synth. Vol. 50, (J. Wiley \& Sons, New York, 1970), pp. 15-18 (m-Chloroperbenzoic acid).

6. L.A. Paquette and J.H. Barrett, In: H.E. Baumgarten (Ed.). Org. Synth Coll. Vol. V, (J. Wiley and Sons, New York, 1973), p. 468 (2,7-Dimethyloxepine).

7. C. Venturello, E. Alneri and M. Ricci, J. Org. Chem. 48, 3831-3833 (1983).

8. J.H. Biel J. Am. Chem. Soc. 71, 1306-1309 (1949).

9. K. Krassuski, J. Prakt. Chem. 75238 (1907). apud. R.E. Parker and N. S. Isaacs, Chem. Rev. 59, 737-799 (1959).

10. K. Leffler and R. Adams, J. Am. Chem. Soc. 59, 2252-2258 (1937)

11. R.E. Parker and N. S. Isaacs, Chem Rev. 59, 2252-2258 (1937).

12. M.T. Leffler and R. Adams, J. Am. Chem. Soc. 59, 2252-2258 (1973).

13. W.W.Y. Liu, D.L. Nelson and D. P. Veloso Ciência e Cultura 35 (supl), 400 (1983).

14. D.L. Klayman and W. F. Gilmore, J. Med. Chem. 7, 823-824 (1064).

15. D.L. Klayman, W. F. Gilmore and T.R. Sweeney, Chem. and Ind. 1632 (1965).

16. D.L. Klayman, J. W. Lown and T.R. Sweeney, J. Org. Chem. 30, 2275-2278 (1965).

17. D.L. Klayman and J.R. Shine, Quart. Rep. Sulfur Chem. 3, 189-316 (1968).

18. E. Block, M.D. Bently, F. A. Davis, I. B. Douglas and J.A. Lacadie, J. Org. Chem.40, 2770-2773 (1975).

19. F. Freeman and C. N. Angeletakis, Org. Mass Spectrom. 17, 114-122 (1982)

20. S.A. Vieira Filho, D.L. Nelson and. D.P. Veloso, $A n$. Acad. brasil. Ciênc. 58, 547-551 (1986).

21. J. Pellegrino and J. Faria, Am. J. Trop. Med. Hyg. 14, 363-369 (1965).

22. J. Pellegrino and N. Katz, In: A. S. Cunha, (Ed), Esquitossomose mansônica (Universidade de São Paulo, São Paulo, 1970), Chapt. VIII pp. 313-326 (Terapêutica Experimental).

23. Aldrich Catalog/Handbook of Fine Chemicals, (Aldrich Chemical Co., Milwaukee, 1984), p. 498.

24. R.T.E. Schenck and S. Kaizerman, J. Am. Chem. Soc. 75, 1636 (1953). 